\title{
Dois anos de Pembrolizumab em monoterapia para o tratamento do carcinoma pulmonar de não-pequenas células, o que se segue? - uma reflexão baseada em dados da prática clínica
}

\section{Two-year treatment with Pembrolizumab monotherapy in advanced non-small cell lung cancer: a reflection based on real-world data}

\author{
Sara Costa-Martins ${ }^{1, *}$ (iD), Salete Valente ${ }^{2}$ (D), Alice Pêgo ${ }^{3}$, Ana Figueiredo ${ }^{3}$ (D), Fernando Barata ${ }^{4}$ (iD) \\ ${ }^{1}$ Interna de Formação Específica em Pneumologia, Serviço de Pneumologia do Centro Hospitalar Universitário Cova da Beira \\ ${ }^{2}$ Assistente Hospitalar Graduada Sénior em Pneumologia, do Serviço de Pneumologia do Centro Hospitalar Universitário Cova \\ da Beira \\ ${ }^{3}$ Assistente Hospitalar Graduada em Pneumologia, Serviço de Pneumologia do Centro Hospitalar e Universitário de Coimbra \\ ${ }^{4}$ Assistente Hospitalar Graduado Sénior em Pneumologia, do Serviço de Pneumologia do Centro Hospitalar e Universitário de \\ Coimbra \\ *sara.m.costamartins@gmail.com
}

\section{RESUMO}

Introdução e objetivos: A imunoterapia tem introduzido avanços no tratamento do carcinoma pulmonar de não-pequenas células. Contudo, dados da prática clínica atual sobre potenciais grupos respondedores, duração ideal de tratamento e questões de segurança permanecem por esclarecer. Pretende-se produzir uma reflexão sobre a população de doentes com resposta favorável ao Pembrolizumab e como têm evoluído clinicamente longo do tempo.

Materiais e métodos: Estudo retrospetivo de doentes que cumpriram pelo menos 2 anos/35 ciclos de Pembrolizumab, com análise descritiva das características clinicopatológicas e dos resultados médicos obtidos ao longo do período de seguimento.

Resultados: Identificaram-se 23 doentes, maioritariamente, homens (95.7\%), diagnosticados com adenocarcinoma (78.3\%) em média aos 62.0anos de idade, apresentando bom estado funcional. Durante o tratamento, $69.6 \%$ dos casos alcançaram resposta objetiva. À data do estudo, com um seguimento mediano de 35.2meses, 17 doentes estavam vivos e em remissão (73.9\%), 7 dos quais (41.1\%) com doença controlada por tempo superior a 32.6 (máximo observado de 48.7)meses; entre os que que progrediram (13.0\%), apenas 1 alcançou novamente resposta parcial ao fim de 5.1 meses desde a reintrodução da imunoterapia. Ocorreram eventos adversos grau >2 em 9 doentes (39\%), condicionando diferentes desfechos clínicos; desses, $44 \%$ evidenciaram reincidência mesmo após suspensão da imunoterapia. 
Discussão e conclusões: Os dados parecem corroborar o potencial benefício duradouro do Pembrolizumab descrito em estudos recentes, com perfil de segurança manejável, embora não desprezível. Na ausência de toxicidade relevante e perante progressão da doença, um segundo curso poderá ser ponderado. Todavia, mais estudos prospetivos e multicêntricos, com maior amostra e longo período de seguimento, são necessários para sustentar estes dados.

Palavras-chave: Carcinoma pulmonar de não-pequenas células, imunoterapia, pembrolizumab, seguimento, prognóstico.

(c) 2021 Grupo de Estudos do Cancro do Pulmão. Publicado por Publicações Ciência \& Vida. Este é um artigo Open Access sob uma licença CC BY-NC-ND (http://creativecommons.org/licenses/by-nc-nd/4.0/).

\begin{abstract}
Introduction and objectives: Immunotherapy has introduced advances in the treatment of non-small cell lung cancer. However, data from current clinical practice on potential responder groups, optimal duration of treatment and safety issues remain unclear. It is intended to produce a reflection on the population of patients with a favourable response to Pembrolizumab and how they have evolved clinically over time.

Materials and methods: Retrospective study of patients who completed at least 2 years/35 cycles of Pembrolizumab was conducted, with descriptive analysis of clinicopathological characteristics and medical results obtained during the follow-up period.

Results: Twenty-three patients were identified, mostly men (95.7\%), diagnosed with adenocarcinoma (78.3\%) at an average age of 62.0 -year-old, with good functional status. During treatment, $69.6 \%$ of cases achieved an objective response. At the time of the study, with a median follow-up of 35.2 months, 17 patients were alive and in remission $(73.9 \%), 7$ of which $(41.1 \%)$ with disease controlled for more than 32.6 months (maximum observed of 48.7 ) months; among those who progressed (13.0\%), only 1 achieved a partial response again within 5.1 months of reintroduction of immunotherapy. Grade $>2$ adverse events occurred in 9 patients (39\%), leading to different clinical outcomes; of these, $44 \%$ showed recurrence even after suspension of immunotherapy.

Discussion and conclusions: The data seem to support the potential lasting benefit of Pembrolizumab described in recent studies, with a manageable, although not negligible, safety profile. In the absence of relevant toxicity and in the face of disease progression, a second course may be considered. However, more prospective, and multicenter studies, with a larger sample and a longer follow-up period, are needed to support these data.
\end{abstract}

Keywords: Non-small cell lung cancer, immunotherapy, pembrolizumab, follow-up, prognosis.

(C) 2021 Grupo de Estudos do Cancro do Pulmão. Published by Publicações Ciência \& Vida. This is an open access article under the CC BY-NC-ND license (http://creativecommons.org/licenses/by-nc-nd/4.0/).

\section{INTRODUÇÃO}

O carcinoma pulmonar de não-pequenas células (CPNPC) representa o tipo mais comum de neoplasia maligna do pulmão, compreendendo
85 a $90 \%$ dos $\operatorname{casos}^{1-3}$. Na sua maioria, não se identificam alterações genéticas alvo de terapêuticas dirigidas e, ao diagnóstico, tendem a apresentar-se em estadios avançados ${ }^{4}$. Os esquemas de quimioterapia contendo platina cons- 
tituem opções terapêuticas sistémicas clássicas, embora com taxas de sobrevida global aos 5 anos inferiores a $7 \%^{2}$. Contudo, nos últimos anos, a investigação de novos agentes, nomeadamente de imunoterapia, tem contribuído para uma meIhoria do prognóstico e, por conseguinte, para uma mudança do paradigma do tratamento destes doentes 5 .

Especificamente no caso do CPNPC metastático sem mutações tratáveis, dados de segurança e eficácia obtidos em ensaios clínicos randomizados suportaram a aprovação de Pembrolizumab em monoterapia em Portugal, tanto em primeira como em segunda ou outras linhas terapêuticas, de acordo com a expressão tumoral de PD-L1 ( $\geq 50 \%$ e $\geq 1 \%$, respetivamente), até à progressão da doença ou desenvolvimento de toxicidade inaceitável ${ }^{6}$. Estudos de seguimento aos três e, mais recentemente, cinco anos têm reforçado os resultados positivos encontrados, demonstrando respostas antitumorais duradouras e benefícios significativos a nível de sobrevivência global e livre de progressão, tolerabilidade e qualidade de vida a longo-prazo ${ }^{7-9}$. No entanto, alguns pontos permanecem por esclarecer:

- Menos de metade dos doentes candidatos para tratamento em monoterapia com base nos valores de PD-L1 beneficiam com o tratamento e atingem os dois anos de imunoterapia, não sendo conhecidos claros fatores preditores de resposta ${ }^{10}$;

- Embora a prática médica atual seja orientada por evidências de ensaios clínicos, resultados em contexto de vida real, referentes ao seguimento dos doentes durante o período subsequente à conclusão dos dois anos do tratamento em questão, acrescentam informações importantes, mas são ainda limitados na literatura ${ }^{11-12}$;
- Não está estabelecida a duração ideal do tratamento com Pembrolizumab e se este pode ser continuado por um período mais longo enquanto o fármaco for tolerado ou mesmo reintroduzido perante progressão tumoral, se tiver sido suspenso antes da progressão da doença ${ }^{13-14}$.

O presente estudo tem como principal objetivo proporcionar uma reflexão sobre a população de doentes com cancro do pulmão em estadio avançado que, respondendo favoravelmente à imunoterapia com Pembrolizumab, conseguem completar pelo menos 35 ciclos ou 2 anos de tratamento (como preconizado nos ensaios clínicos randomizados que suportaram a sua aprovação), e sobre a respetiva evolução clínica ao longo do período subsequente, tendo por base a análise de resultados da prática médica regular.

\section{MATERIAL E MÉTODOS}

Foi desenvolvido um estudo retrospetivo envolvendo todos os doentes seguidos por CPNPC em consulta de Pneumologia Oncológica de dois hospitais (Centro Hospitalar e Universitário de Coimbra e Centro Hospitalar Universitário Cova da Beira) que, até 1 de setembro de 2021, cumpriram pelo menos 35 ciclos ou 2 anos de tratamento com Pembrolizumab $200 \mathrm{mg}$ a cada $3 \mathrm{se}-$ manas ou $400 \mathrm{mg}$ a cada 6 semanas por via endovenosa.

Após identificação da população a caracterizar, foram recolhidos, através de consulta dos registos hospitalares informatizados, dados demográficos e clinicopatológicos ao diagnóstico, bem como resultados clínicos referentes ao período de tempo desde o início de Pembrolizumab até à data da última avaliação (morte ou data- 
-limite do estudo, 01 de setembro de 2021), definindo-se este como o período de seguimento do doente. Foram extraídas informações sobre a resposta inicial ao tratamento e duração da mesma, terapêuticas subsequentes e incidência de efeitos adversos relacionados com a imunoterapia de grau superior a 2. A definição de progressão da doença teve por base uma avaliação segundo os critérios de Response Evaluation Criteria in Solid Tumors (RECIST v1.1).

Foi realizada uma análise descritiva dos dados globais da coorte em estudo e, ainda, tendo em consideração a estratificação por linha de tratamento instituída (primeira, zsegunda linha). Para além de tabelas, os resultados foram apresentados sob a forma de gráfico de modo a tornar visualmente percetível a evolução de cada doente ao longo do período de seguimento.

O tratamento estatístico foi realizado com recurso ao software IBM ${ }^{\circledR}$ SPSS 20.

\section{RESULTADOS}

O presente estudo reuniu uma amostra constituída por 23 doentes que iniciaram tratamento com Pembrolizumab em monoterapia entre fevereiro de 2017 e agosto de 2019, 43\% dos quais em primeira linha terapêutica, enquanto os restantes teriam cumprido previamente outros regimes sistémicos com quimioterapia.

A Tabela I apresenta as características basais do total de doentes incluídos e agrupados segundo a linha de tratamento instituída no momento de início da imunoterapia. Eram, maioritariamente, doentes do género masculino $(95.7 \%)$, com história de tabagismo (91.3\%) e que, ao diagnóstico, apresentavam idade média de 62.0 anos (intervalo de 40 a 81 anos) e performance status entre 0 e 1 segundo a escala Eastern Cooperative
Oncology Group (ECOG). Entre os doentes com comorbilidades associadas e necessidade de respetiva medicação crónica (65.2\%), o grupo de patologias cardiovasculares foi o mais prevalente $(80 \%)$, seguido das metabólicas $(30.4 \%)$, neoplásicas $(20 \%)$ e, em igual proporção $(13.3 \%)$ das pulmonares e autoimunes. O tipo histológico mais frequente era o adenocarcinoma, todos com expressão positiva de PD-L1, estando o respetivo valor em concordância com a decisão de cada linha terapêutica; 21 dos casos (91.3\%) correspondiam a estadios metastáticos desde o início do tratamento, embora a maioria sem envolvimento do sistema nervoso central.

À data do estudo, o período de seguimento mediano do total de doentes analisados no estudo era de 35.2 (24.2-54.2) meses desde o início do tratamento e 11.0 meses (1.4-27.1 meses) desde a sua suspensão. $O$ subgrupo que já tinha recebido tratamento prévio com quimioterapia apresentou maior tempo de seguimento em relação ao de primeira linha terapêutica (40.9 vs. 30.8 meses).

Na Tabela II encontram-se sumariados dados que refletem a resposta ao tratamento com Pembrolizumab. No global, os doentes mantiveram-se em tratamento durante uma mediana de 24.9 meses e 35 ciclos, até um máximo de 33.5 meses e 47 ciclos. Foi alcançada resposta objetiva (completa ou parcial) em 16 casos (69.6\%), ao fim de um tempo mediano de 3.0 meses desde o início do tratamento, de forma similar entre os dois grupos representados na Tabela II; 12 destes doentes $(75.0 \%)$ mantiveram continuamente a resposta, sem desfechos adversos.

A Figura 1 sistematiza os resultados clínicos dos doentes durante todo o período de seguimento.

Em setembro de 2021, 3 casos (13.0\%) tinham evoluído com progressão da doença ao fim de

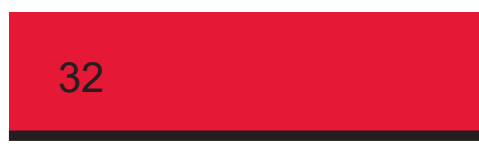


Tabela I. Características demográficas e clínicas dos doentes ao início do tratamento com Pembrolizumab.

\begin{tabular}{|c|c|c|c|}
\hline & $\begin{array}{l}\text { Total } \\
(n=23)\end{array}$ & $\begin{array}{c}1 .{ }^{a} \text { linha } \\
(n=10)\end{array}$ & $\begin{array}{c}\geq 2 .^{a} \text { linha } \\
\quad(n=13)\end{array}$ \\
\hline \multicolumn{4}{|l|}{ Género } \\
\hline Masculino & $22(95.7)$ & $10(100.0)$ & $12(92.3)$ \\
\hline Feminino & $1(4.3)$ & 0 & $1(7.7)$ \\
\hline \multicolumn{4}{|l|}{ Grupo etário } \\
\hline$<65$ & $14(60.9)$ & $5(50.0)$ & $9(69.2)$ \\
\hline$\geq 65$ & $9(39.1)$ & $5(50.0)$ & $4(30.8)$ \\
\hline Idade mediana, anos & $63(40-81)$ & $66(47-81)$ & $59(40-79)$ \\
\hline \multicolumn{4}{|l|}{ História de tabagismo } \\
\hline Nunca fumador & $2(8.7)$ & 0 & $2(15.4)$ \\
\hline Ex-fumador & $8(34.8)$ & $3(30.0)$ & $5(38.5)$ \\
\hline Fumador ativo & $13(56.5)$ & $7(70.0)$ & $6(46.2)$ \\
\hline \multicolumn{4}{|l|}{ Comorbilidades } \\
\hline Sim & $15(65.2)$ & $9(90.0)$ & $6(46.2)$ \\
\hline Não & $8(34.8)$ & $1(10.0)$ & $7(53.8)$ \\
\hline \multicolumn{4}{|l|}{ ECOG performance status } \\
\hline 0 & $3(13.0)$ & $1(10.0)$ & $2(15.4)$ \\
\hline 1 & $20(87.0)$ & $9(90.0)$ & $11(84.6)$ \\
\hline$\geq 2$ & 0 & 0 & 0 \\
\hline \multicolumn{4}{|l|}{ Histologia } \\
\hline Adenocarcinoma & $18(78.3)$ & $8(80.0)$ & $10(76.9)$ \\
\hline Escamoso & $2(8.7)$ & 0 & $2(15.4)$ \\
\hline Adenoescamoso & $1(4.3)$ & 0 & $1(7.7)$ \\
\hline Outro & $2(8.7)$ & $2(20.0)$ & 0 \\
\hline \multicolumn{4}{|l|}{ Expressão de PD-L1 } \\
\hline $1-49$ & $6(26.1)$ & 0 & $6(46.2)$ \\
\hline$\geq 50$ & $17(73.9)$ & $10(100.0)$ & $7(53.8)$ \\
\hline \multicolumn{4}{|l|}{ Metastização cerebral } \\
\hline Não & $19(82.6)$ & $9(90.0)$ & $10(76.9)$ \\
\hline Sim & $4(17.4)$ & $1(10.0)$ & $3(23.1)$ \\
\hline
\end{tabular}

n - número de doentes (\%)

um intervalo de tempo que variou entre 3.6 e 22.2 meses após a suspensão de Pembrolizumab, o qual foi prescrito em todos como segunda linha de tratamento. Estes tinham obtido resposta par-
Tabela II. Resposta ao tratamento com Pembrolizumab.

\begin{tabular}{|c|c|c|c|}
\hline & $\begin{array}{l}\text { Total } \\
(n=23)\end{array}$ & $\begin{array}{l}\text { 1. }^{\mathrm{a}} \text { linha } \\
(n=10)\end{array}$ & $\begin{array}{c}\geq 2 .^{a} \text { linha } \\
\quad(n=13)\end{array}$ \\
\hline $\begin{array}{l}\text { Taxa de resposta } \\
\text { objetiva, \% }\end{array}$ & 69.6 & 70.0 & 69.2 \\
\hline Melhor resposta obtida & & & \\
\hline Resposta completa & $1(4.3)$ & $1(10.0)$ & 0 \\
\hline Resposta parcial & $15(65.2)$ & $6(60.0)$ & $9(69.2)$ \\
\hline Doença estável & $7(30.4)$ & $3(30.0)$ & $4(30.8)$ \\
\hline $\begin{array}{l}\text { Tempo mediano até } \\
\text { resposta, meses }\end{array}$ & $\begin{array}{c}3.0 \\
(2.0-17.2)\end{array}$ & $2.7(2.1-5.3)$ & $\begin{array}{c}3.4 \\
(2.0-17.2)\end{array}$ \\
\hline $\begin{array}{l}\text { Duração mediana de } \\
\text { resposta, meses }\end{array}$ & $\begin{array}{c}33.1 \\
(19.6-n a)\end{array}$ & $\begin{array}{c}27.1 \\
(19.6-n a)\end{array}$ & $\begin{array}{c}37.9 \\
(25.3-n a)\end{array}$ \\
\hline $\begin{array}{l}\text { Doença progressiva, n } \\
\text { (\%) }\end{array}$ & $3(13.0 \%)$ & 0 & $3(23.1 \%)$ \\
\hline
\end{tabular}

n- número de doentes (\%); na - não atingida.

cial $(n=1)$ e doença estável $(n=2)$ com o primeiro curso de imunoterapia. Um segundo curso com o mesmo agente terapêutico foi tentado em 2 doentes deste grupo, mas apenas 1 alcançou novamente resposta parcial ao fim de 5.1 meses desde a data de progressão de doença.

Registaram-se 4 óbitos (17.4\%), 2 na sequência do desenvolvimento de outra neoplasia maligna síncrona extratorácica, 1 por progressão da doença apesar de tentativa de um segundo curso de Pembrolizumab e 1 no contexto de toxicidade pulmonar grave imunomediada, desenvolvida após 40 ciclos de tratamento.

Dos 19 doentes vivos durante o período analisado (82.6\%), 17 (89.5\%) permaneceram em vigilância ativa, sem evidência de progressão tumoral; 7 destes casos (41.1\%) tinham concluído a imunoterapia há pelo menos 12 meses e apresentavam longas durações de doença controlada, superiores a 32.6 (mediana 37.9, máximo observado 48.7) meses. Quando avaliada retrospetivamente a melhor resposta 
obtida durante o tratamento, todos tinham apresentado resposta parcial, 3 dos quais tinham cumprido mais de dois anos de imunoterapia por opção.

Ao longo do período avaliado, foram reportados eventos adversos com necessidade de corticoterapia ou mesmo interrupção temporária de imunoterapia em 9 doentes durante o tratamento com Pembrolizumab (39.1\%). A idade mediana deste grupo era de 66 (50-81) anos, todos apresentavam performance status ECOG 1 e, na glo- balidade, reuniam outras patologias crónicas $(77.7 \%)$; a resposta objetiva foi alcançada em $77.7 \%$ dos casos. A maioria tinha desenvolvido mais do que um efeito isolado, com atingimento de diferentes sistemas: artrite $(n=6)$, pneumonite $(n=3)$, colite $(n=2)$, dermatite $(n=2)$, tiroidite $(n=$ 1). Em $44 \%(n=4)$ destes casos, houve reincidência após suspensão terapêutica ao final dos 2 anos (registando-se pneumonite $(n=2)$, artrite $(n=2)$, colite $(n=1))$, como se observa no gráfico da Figura 1.

Figura 1. Resultados clínicos dos doentes que completaram 35 ciclos ou 2 anos de tratamento com Pembrolizumab, apresentados ao longo do tempo, em meses. As barras escuras representam o período de tempo sob imunoterapia, seguidas pelas barras claras que exibem o período subsequente até à data da última avaliação (morte ou data-limite do estudo).

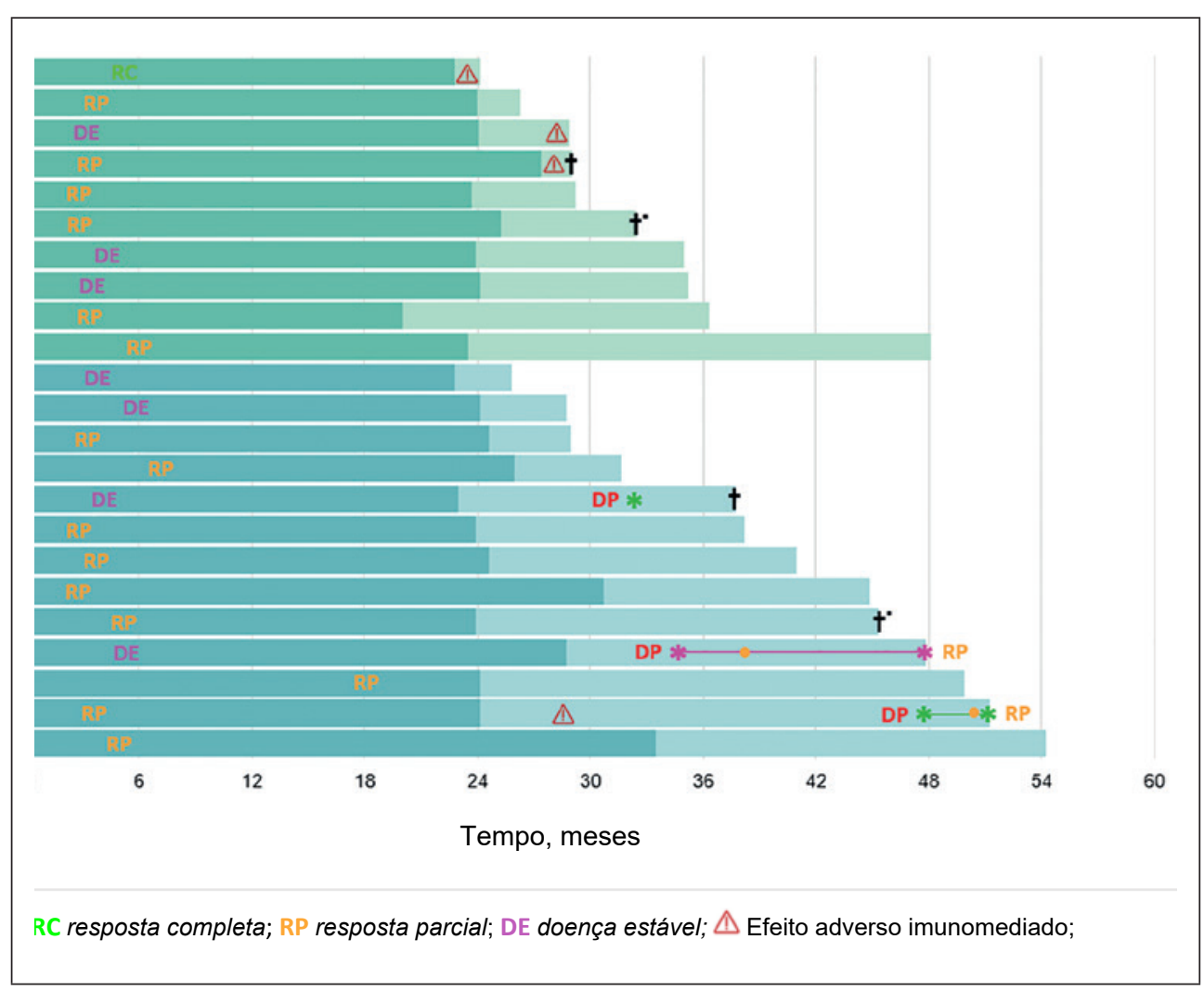

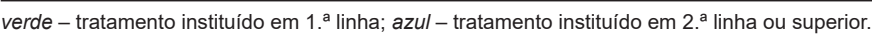


Aqueles doentes que não desenvolveram toxicidade relevante durante o período inicial de tratamento, não desenvolveram igualmente efeitos laterais tardios após suspensão do fármaco. Em confrontação, este grupo de doentes tinha idade mediana inferior (59.5 (40-79) anos), menos comorbilidades (57.1\%) e incluía, também, doentes com ECOG 0; nestes, a resposta objetiva foi atingida em $64.3 \%$.

\section{DISCUSSÃO}

Este trabalho pretende produzir uma reflexão sobre a experiência clínica com doentes diagnosticados com CPNPC metastático que cumpriram um primeiro curso de tratamento com Pembrolizumab, apesar das limitações que se reconhecem como inerentes a este tipo de estudo.

Foram identificados todos os doentes com CPNPC metastático que, à luz das evidências de ensaios clínicos e consequentes atualizações das linhas de orientação terapêutica, completaram pelo menos 2 anos/ 35 ciclos de Pembrolizumab em monoterapia. Em Portugal, este regime terapêutico foi primeiramente aprovado para doentes com expressão de PD-L1 $\geq 1 \%$ e que tivessem recebido pelo menos um esquema de tratamento prévio com quimioterapia e, mais tarde, em primeira linha naqueles com PD-L1 $\geq 50 \%{ }^{6}$. Este facto, justifica a diferença a nível do tempo de seguimento observado entre os dois subgrupos (40.9 vs. 30.8 meses), não sendo possível, por isso, efetuar um estudo comparativo dos resultados a longo-prazo encontrados em cada grupo, por enquanto.

De um modo geral, as características clinicopatológicas dos doentes avaliados assemelham-se às descritas na literatura para o CPNPC, à exceção da menor percentagem de doentes no grupo etário acima dos 65 anos, tal como foi encontrado na população de doentes que completou 35 ciclos/ 2 anos de Pembrolizumab do ensaio Keynote- $010^{15}$.

Globalmente, os doentes cumpriram a duração preconizada para a imunoterapia, com exceção de três que, perante resposta parcial mantida, prolongaram para além dos 35 ciclos após decisão partilhada entre o médico e o doente, embora com resultados clínicos distintos entre si: 1 faleceu no contexto de toxicidade pulmonar grave reincidente, enquanto os outros, sem história de eventos adversos prévios, evoluíram até à data do estudo sem intercorrências. Estes casos específicos ilustram como o risco de exposição contínua ao tratamento com anti-PD-1 e o respetivo perfil de segurança pode ser variável de indivíduo para indivíduo, devendo, por isso, ser considerados quando se pondera prolongar o tratamento. Além disso, a questão de reintroduzir o fármaco após evento adverso significativo é também relevante, embora ainda não se encontre completamente explorada em dados prospetivos de ensaios clínicos ${ }^{14,15}$. Um estudo que avaliou a reintrodução de anticorpo anti-PD-1/ PD-L1 em 38 doentes com CPNPC que já tinham desenvolvido um evento adverso imunomediado grave verificou que $48 \%$ evoluiu sem novas ocorrências, $26 \%$ teve uma recorrência do evento inicial e $26 \%$ apresentou um novo evento ${ }^{16}$. Estes dados parecem reforçar a ideia de que a duração ideal do tratamento seja baseada em informações individuais, como: gravidade dos efeitos imunomediados anteriores, performance status do doente, disponibilidade de tratamentos alternativos e resposta inicial observada durante o tratamento.

Ainda a propósito de dados de segurança, a incidência de eventos adversos significativos relacionados com o tratamento, neste estudo, foi superior à descrita na literatura (relatos com va- 
lores a rondar os $20 \%{ }^{14}$ ). Os dados encontrados englobam, mais frequentemente, doentes de faixa etária superior e com outras patologias crónicas associadas, contudo, dizem respeito a uma reduzida série, necessitando de mais validação. De forma congruente com outros achados descritos na literatura, os efeitos adversos tardios/ reincidentes, embora mais raros, verificaram-se entre o grupo de doentes que já haviam relatado toxicidade anteriormente sob tratamento com Pembrolizumab, pelo que uma vigilância ativa com identificação precoce de sinais e sintomas relacionados deve ser mantida mesmo após cessação do tratamento para uma intervenção oportuna, muitas vezes desafiante ${ }^{14}$.

Relativamente aos resultados clínicos observados, a maioria alcançou resposta objetiva com o Pembrolizumab em monoterapia, de forma célere, dentro do intervalo de tempo expectável para este grupo de respondedores à imunoterapia, e duradoura ${ }^{7}$. Além disso, esses doentes, em comparação com os que apresentaram estabilidade segundo os critérios RECIST, progrediram mais lentamente. Quando estudado especificamente o subgrupo de doentes com período de seguimento superior a 12 meses, são também evidentes os benefícios a nível de sobrevivência associados aos doentes com melhor resposta inicial ao tratamento. Entre os casos de doença progressiva, destaca-se um em que, 22 meses após a suspensão do Pembrolizumab, foi possível reiniciar-se um segundo curso de tratamento com o mesmo agente, com novo benefício sustentado. Embora se trate de uma amostra reduzida, estes dados são concordantes com os estudos a longo-prazo dos ensaios clínicos Keynote-001, -010 e -024 , que conduziram às guidelines internacionais atuais $^{7-9}$.

Em resumo, os resultados apresentados refletem o contexto real em que decorre a prática médica. Este facto constitui um ponto de destaque deste estudo, pois a maioria dos dados existentes sobre esta temática na literatura dizem respeito a populações de ensaios clínicos, selecionadas, inseridas num ambiente controlado, diferindo, de certa forma, da população heterogénea encontrada na rotina médica, em que a abordagem clínica é muitas vezes influenciada por fatores externos (socioeconómicos, preferências do doente e respetiva adesão ao tratamento $)^{17}$. No entanto, o facto de se tratar de um estudo retrospetivo, e o reduzido tamanho da população, surgem como limitações do ponto de vista de significado estatístico. As diferenças de amplitude do período de seguimento dos doentes analisados também contribuíram de forma limitante para a interpretação dos resultados encontrados, motivo pelo qual a análise descritiva foi acompanhada pela exposição de casos específicos que mereciam maior destaque.

\section{CONCLUSÃO}

Os dados deste estudo traduzem um contexto de mundo real em que decorre a prática clínica de dois serviços dedicados à Pneumologia Oncológica, e revelam o potencial benefício duradouro do Pembrolizumab em doentes com ou sem tratamento prévio com outros regimes terapêuticos sistémicos. Na ausência de toxicidade relevante e perante progressão da doença, caso o fármaco tenha sido suspenso ainda com resposta à terapêutica, um segundo curso poderá ser ponderado, embora ainda seja escasso o tempo de seguimento e a experiência clínica nesse âmbito. O perfil de segurança deste fármaco parece manejável na maioria dos casos, contudo, não desprezível, devendo, sempre, justificar uma atenta vigilância e monitorização, de forma indi-

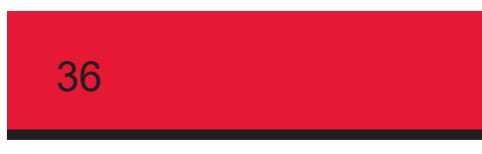


vidualizada, pois os riscos da exposição contínua a esta classe farmacológica parecem ser relevantes e conduzir a desfechos negativos em determinados doentes.

Permanecem ilações limitadas sobre possíveis associações entre as características basais dos doentes e a eficácia de Pembrolizumab, bem como claros preditores que auxiliem na previsão de maior risco de progressão de doença e de desenvolvimento de efeitos adversos imunomediados. Estes achados necessitam de mais sustentação, pelo que deverão motivar a realização de mais estudos prospetivos e multicêntricos, com maior dimensão de amostra e mais longo período de seguimento.

Conflito de interesses: Não existe qualquer conflito de interesses.

\section{ORCID}

\author{
Sara Martins (iD 0000-0002-8216-5452 \\ Salete Valente (iD 0000-0001-9281-2003 \\ Ana Figueiredo (iD 0000-0002-7949-8440 \\ Fernando Barata (iD 0000-0002-6306-0938
}

\section{REFERÊNCIAS}

1. Molina JR, Yang P, Cassivi SD, Schild SE, Adjei AA. Non-small cell lung cancer: epidemiology, risk factors, treatment, and survivorship. Mayo Clin Proc. 2008;83(5):584-594. doi:10.4065/83.5.584

2. Rossi A, Di Maio M. Platinum-based chemotherapy in advanced non-small-cell lung cancer: optimal number of treatment cycles. Expert Rev Anticancer Ther. 2016;16(6):653-660. doi:10.1586/14737140 .2016.1170596

3. Li F, Dong X. Pembrolizumab provides long-term survival benefits in advanced non-small cell lung cancer: The 5-year outcomes of the KEYNOTE-024 trial. Thorac Cancer. 2021;12(23):3085-3087. doi:10.1111/1759-7714.14193

4. Hanna NH, Schneider BJ, Temin S, et al. Therapy for Stage IV Non-Small-Cell Lung Cancer Without Driver Alterations: ASCO and $\mathrm{OH}$ (CCO) Joint Guideline Update. J Clin Oncol 2020; 38:1608.

5. Wood DE, Kazerooni EA, Baum SL, Eapen GA, Ettinger DS, Hou L, et al. Lung cancer screening, version 3.2018, NCCN clinical practice guidelines in oncology. J Natl Compr Canc Netw. 2018;16(4):412-41.

6. European Medicines Agency/703455/2021. Keytruda (pembrolizumab). An overview of Keytruda and why it is authorised in the EU. https://www.ema. europa.eu/en/documents/overview/keytruda-epar-medicine-overview_en.pdf. Acedido em 11/2021.

7. Garon EB, Hellmann MD, Rizvi NA, et al. Five-Year Overall Survival for Patients With Advanced NonSmall-Cell Lung Cancer Treated With Pembrolizumab: Results From the Phase I KEYNOTE-001 Study. J Clin Oncol. 2019;37(28):2518-2527. doi:10.1200/JCO.19.00934

8. Herbst RS, Garon EB, Kim DW, et al. Five Year Survival Update From KEYNOTE-010: Pembrolizumab Versus Docetaxel for Previously Treated, Programmed Death-Ligand 1-Positive Advanced NSCLC. J Thorac Oncol. 2021;16(10):1718-1732. doi:10.1016/j.jtho.2021.05.001

9. Reck M, Rodríguez-Abreu D, Robinson AG, et al. Five-Year Outcomes With Pembrolizumab Versus Chemotherapy for Metastatic Non-Small-Cell Lung Cancer With PD-L1 Tumor Proportion Score $\geq 50$. J Clin Oncol. 2021;39(21):2339-2349. doi:10.1200/ JCO.21.00174

10. Garon EB, Rizvi NA, Hui R, et al. Pembrolizumab for the treatment of non-small-cell lung cancer. $N$ Engl J Med. 2015;372(21):2018-2028. doi:10.1056/ NEJMoa1501824

11. Cramer-van der Welle CM, Verschueren MV, Tonn $\mathrm{M}$, et al. Real-world outcomes versus clinical trial results of immunotherapy in stage IV non-small cell lung cancer (NSCLC) in the Netherlands. Sci Rep. 2021;11(1):6306. doi:10.1038/s41598-021-85696-3

12. Cramer-van der Welle CM, Peters BJM, Schramel $\mathrm{FMNH}$, et al. Systematic evaluation of the efficacy-effectiveness gap of systemic treatments in me- 
tastatic nonsmall cell lung cancer. Eur Respir J. 2018;52(6):1801100. doi:10.1183/13993003.0110 0-2018

13. Herbst RS, Garon EB, Kim DW, et al. Long-term follow-up in the KEYNOTE-010 study of pembrolizumab (pembro) for advanced NSCLC, including in patients (pts) who completed 2 years of pembro and pts who received a second course of pembro. Annals of Oncology. 2018;29(8):749 doi:10.1093/ annonc/mdy424

14. Marron TU, Ryan AE, Reddy SM, et al. Considerations for treatment duration in responders to immune checkpoint inhibitors. J Immunother Cancer. 2021;9(3):e001901. doi:10.1136/jitc-2020-001901

15. Herbst RS, Garon EB, Kim DW, et al. Long-Term Outcomes and Retreatment Among Patients With
Previously Treated, Programmed Death-Ligand 1-Positive, Advanced Non-Small-Cell Lung Cancer in the KEYNOTE-010 Study. J Clin Oncol. 2020;38(14):1580-1590. doi:10.1200/JCO.19. 02446

16. Metro G, Signorelli D. Immune checkpoints inhibitors rechallenge in non-small-cell lung cancer: different scenarios with different solutions?. Lung Cancer Manag. 2020;8(4):LMT18. Published 2020 Jan 16. doi:10.2217/Imt-2019-0012

17. Velcheti V, Chandwani S, Chen X, Pietanza MC, Piperdi B, Burke T. Outcomes of first-line pembrolizumab monotherapy for PD-L1-positive (TPS $\geq 50 \%$ ) metastatic NSCLC at US oncology practices. Immunotherapy. 2019;11(18):1541-1554. doi:10.2217/imt-2019-0177 